\title{
Frequency of Aspirin Misuse among Hypertensive Patients in Babylon Province
}

\author{
Zahraa Fattah Ghani ${ }^{1}$, Oday Jasim Alsalihi ${ }^{2}$, Mustafa Shakir Mahmood ${ }^{3}$ \\ ${ }^{1}$ Marjan Medical City/Iraq, ${ }^{2}$ College of Medicine - University of Babylon/Iraq, ${ }^{3}$ Ministry of Health/Iraq
}

\begin{abstract}
Background: Hypertension endangering a risk of cardiovascular events and BP reduction will reduce such complications. the role of aspirin in hypertension is debated, however it is advised in primary prevention in case of patient with high risk factors assessed by one of the cardiovascular risk calculator, a state in which the benefit of aspirin outweigh the risk of complications.
\end{abstract}

Objectives: Estimate the prevalence of aspirin misuse in hypertensive patients, which population at risk and estimate the possible source of this misuse habit.

Patients and Methods: A cross sectional study that has been conducted in babil city major hospitals for the period from the $20^{\text {th }}$ of January to 2 nnd of May. our study sample included 353 patients all cases were between 35 to 75 years old who attend to internal medicine outpatient clinic, emergency departments and inpatients of the above hospitals . All patient had hypertension being treated with antihypertensive agents and they did not experience any vascular events like CVA, IHD and PVD.

Results: $30.59 \%$ of patient do not use aspirin and $64.31 \%$ of patients use aspirin prescribed by doctors. Most of aspirin use was according to doctor advice( 92.7\%). 5.1 a use aspirin by advice from others. Aspirin use was more with increased age group ( $p$ value 0.003 ) and in females( $p$ value is 0.04 ). No significant differences between educational level, economic status, residency and occupation with the use of aspirin. Regarding aspirin intake by their doctors and score $<10 \%$ : women were more than men ,age $40-60$ years. Mostly were urban, higher education of 50\% had positive history of CVD. Patient who need aspirin but they didn't use aspirin, were 66 patients mostly male live in urban area higher education, smoker , most of them had no family history of cardiovascular diseases.

Conclusion: Aspirin use requires coordinated interaction between patient and physician with encouraging the importance patient education about aspirin use, side effects and CVD risk and proper evaluation of patients risk factors and stratification using simplified charts accessible in health centers.

Key words: Aspirin, primary prevention, hypertension

\section{Introduction}

The CVD risk associated with hypertension depend on multiple factors including age, gender, in addition to BW, physical activity, cigarrete smoking, family history of hypertension, high cholesterol level, DM and preexisting vascular disease ${ }^{(1)}$. Assessment of the risk of future CVD is an important step in patients requiring primary protection from any vascular events ${ }^{(2)}$. However, to determine the role of aspirin's in prevention of CVD, both primarily and secondarily the benefits should be balanced against the complications ${ }^{(3)}$. The net benefit of aspirin in patients with known risk factors is clearly evident ${ }^{(4)}$. For primary protection ,guidelines vary about whether, and to which groups, aspirin is to be given $^{(5)}$ in that context, physicians should evaluate the risks and benefits of aspirin therapy for those patients free of risk factors ${ }^{(6)}$

Primary cardiovascular $(\mathrm{CV})$ prevention defined as the use of pharmacological and/or nonpharmacological measures as prophylaxis of atherosclerosis to prevents 
MCEs in in the absence of any history or clinical signs of underlying disease. The main outcomes to be measured are MI, stroke, and death from CVD. In addition to other outcomes including hospital admission, intervention of coronary disease, incident angina, all-cause mortality are sometimes added as ancillary end points these are applied to apparently healthy persons ${ }^{(7)}$.

For the calculation of an individual probability to develop CVD all factors that contribute to the risk must be taken into account, including previous CVD events ${ }^{(8)}$. Many risk calculators are currently available that help in the detection of population at risk of developing $\mathrm{CHDs}^{(9)}$.

\section{Patients and Method}

A cross sectional descriptive study was conducted in Babylon city hospitals including al hila teaching hospital, marjan medical city and imam al sadiq teaching hospital. For the period from the $20^{\text {th }}$ of January to $2 \mathrm{nnd}$ of May. This study has been done on patients diagnosed by their doctor having high blood pressure and take medication for it.

The data was collected using a preformed questionnaire by which the patient was interviewed after giving appropriate verbal consent .Each patient was interviewed for about 15 minutes

The questionnaire include the following Parts : Patient sosciodemographic characters (age, gender, residency, educational level, economic status, occupation and marital status, the parameters related to 10 years cardiovascular risk score, the protocol of study was approved by ethical committee of Babylon university, college of medicine. Appropriate verbal consent was taken from the patients before the interview. Confidentiality of patients data was approved by replacing names by numbers. To accomplish the study official agreement was taken from Babylon health directorate.

\section{Results}

Table (1) shows the sociodemogrphic distribution of the study sample and the association with the habit of taking aspirin. The association between the age group and the use of aspirin was significant in which $p$ value was 0.003 . In which aspirin use was more with increased age group, also the association between gender and use of aspirin was significant when aspirin use was more in females $\mathrm{p}$ value is 0.04 .

Table (1): The association between sociodeogrphic characteristics of the patients and aspirin taking.

\begin{tabular}{|c|c|c|c|c|}
\hline Characteristic & Group & Use Aspirin & Not users & $P$ value \\
\hline \multirow{3}{*}{ Age } & up to 40 years & $4(80.0 \%)$ & $1(20.0 \%)$ & \multirow{3}{*}{$0.003 *$} \\
\hline & $41-60$ years & $144(63.2 \%)$ & $84(36.8 \%)$ & \\
\hline & more than 60 years & $97(80.8 \%)$ & $23(19.2 \%)$ & \\
\hline \multirow{2}{*}{ Gender } & male & $159(66.0 \%)$ & $82(34.0 \%)$ & \multirow{2}{*}{$0.04 *$} \\
\hline & female & $86(76.8 \%)$ & $26(23.2 \%)$ & \\
\hline \multirow{2}{*}{ Residency } & urban & $206(69.1 \%)$ & $92(30.9 \%)$ & \multirow{2}{*}{0.792} \\
\hline & rural & $39(70.9 \%)$ & $16(29.1 \%)$ & \\
\hline \multirow{2}{*}{ Education } & no or primary education & $74(68.5 \%)$ & $34(31.5 \%)$ & \multirow{2}{*}{0.810} \\
\hline & $\begin{array}{l}\text { medium or high } \\
\text { education }\end{array}$ & $171(69.8 \%)$ & $74(30.2 \%)$ & \\
\hline \multirow{3}{*}{ Occupation } & employed & $98(65.3 \%)$ & $52(34.7 \%)$ & \multirow{3}{*}{0.157} \\
\hline & worker & $66(68.0 \%)$ & $31(32.0 \%)$ & \\
\hline & no job & $81(76.4 \%)$ & $25(23.6 \%)$ & \\
\hline \multirow{3}{*}{ Economic status } & good & $192(66.9 \%)$ & $95(33.1 \%)$ & \multirow{3}{*}{0.051} \\
\hline & fair & $46(78.0 \%)$ & $13(22.0 \%)$ & \\
\hline & not enough & $7(100.0 \%)$ & $0(.0 \%)$ & \\
\hline
\end{tabular}




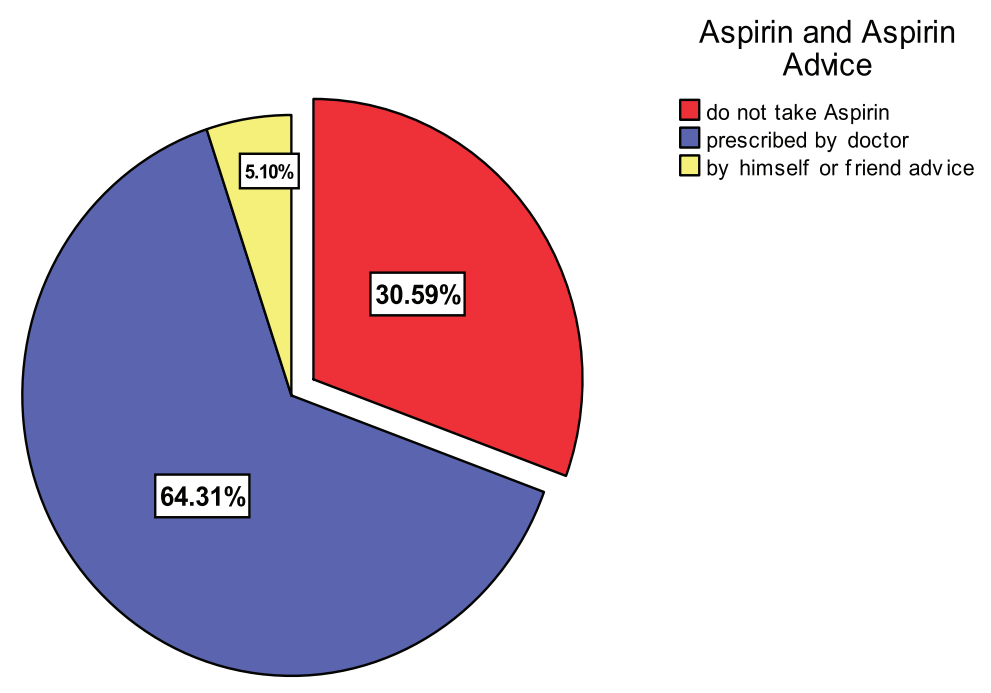

Figure (1): Distribution of Aspirin prescription in total sample

Table(2) Sociodemographic distribution of aspirin misuse by doctor prescription.

\begin{tabular}{|c|c|c|c|c|c|}
\hline \multicolumn{2}{|c|}{ Characteristic } & Group & frequency & percent & total \\
\hline \multirow{2}{*}{\multicolumn{2}{|c|}{ Age }} & $41-60$ years & 24 & 63.2 & \multirow{19}{*}{38} \\
\hline & & more than 60 years & 14 & 36.8 & \\
\hline \multirow{2}{*}{\multicolumn{2}{|c|}{ Gender }} & male & 18 & 47.4 & \\
\hline & & female & 20 & 52.6 & \\
\hline \multirow{2}{*}{\multicolumn{2}{|c|}{ Residency }} & urban & 21 & 55.3 & \\
\hline & & rural & 17 & 44.7 & \\
\hline \multirow{2}{*}{\multicolumn{2}{|c|}{ Education group }} & no or primary education & 17 & 44.7 & \\
\hline & & high education & 21 & 55.3 & \\
\hline \multirow{3}{*}{\multicolumn{2}{|c|}{ Occupation }} & employed & 9 & 23.7 & \\
\hline & & worker & 14 & 36.8 & \\
\hline & & no job & 15 & 39.5 & \\
\hline \multirow{2}{*}{\multicolumn{2}{|c|}{$\begin{array}{l}\text { Marital } \\
\text { status }\end{array}$}} & married & 29 & 76.3 & \\
\hline & & single & 9 & 23.7 & \\
\hline \multirow{2}{*}{\multicolumn{2}{|c|}{ Family history of hypertension or other CVD }} & 19 & 50.0 & 50.0 & \\
\hline & & 19 & 50.0 & 50.0 & \\
\hline \multirow[t]{2}{*}{ Diabetic } & yes & 8 & 21.1 & 21.1 & \\
\hline & no & 30 & 78.9 & 78.9 & \\
\hline \multirow{2}{*}{ smoker } & yes & 5 & 13.2 & 13.2 & \\
\hline & no & 33 & 86.8 & 86.8 & \\
\hline
\end{tabular}


Table(3)Sociodemographic distribution of aspirin misuse by patients.

\begin{tabular}{|c|c|c|c|c|c|c|}
\hline \multicolumn{2}{|c|}{ Characteristic } & \multicolumn{2}{|c|}{ Group } & frequency & percent & total \\
\hline \multirow{2}{*}{\multicolumn{2}{|c|}{ Age }} & \multicolumn{2}{|c|}{ Up to 40 years } & 4 & 44.4 & \multirow{14}{*}{9} \\
\hline & & \multicolumn{2}{|c|}{ 41-60 years } & 5 & 55.6 & \\
\hline \multirow{2}{*}{\multicolumn{2}{|c|}{ Gender }} & \multicolumn{2}{|c|}{ male } & 5 & 55.6 & \\
\hline & & \multicolumn{2}{|c|}{ female } & 4 & 44.4 & \\
\hline \multicolumn{2}{|c|}{ Residency } & \multicolumn{2}{|c|}{ urban } & 9 & 100.0 & \\
\hline \multicolumn{2}{|c|}{ Educational level } & \multicolumn{2}{|c|}{ high education } & 9 & 100.0 & \\
\hline \multicolumn{2}{|c|}{ Occupation } & \multicolumn{2}{|c|}{ employed } & 9 & 100.0 & \\
\hline \multirow{2}{*}{\multicolumn{2}{|c|}{$\begin{array}{l}\text { Marital } \\
\text { status }\end{array}$}} & \multicolumn{2}{|c|}{ married } & 7 & 77.8 & \\
\hline & & \multicolumn{2}{|c|}{ single } & 2 & 22.2 & \\
\hline \multicolumn{2}{|c|}{ Family history of hypertension or other CVD } & yes & 9 & \multicolumn{2}{|l|}{100.0} & \\
\hline \multirow[t]{2}{*}{ Diabetic } & yes & \multicolumn{2}{|l|}{7} & \multirow{2}{*}{\multicolumn{2}{|c|}{$\begin{array}{l}77.8 \\
22.2\end{array}$}} & \\
\hline & no & \multicolumn{2}{|l|}{2} & & & \\
\hline \multirow{2}{*}{ smoker } & yes & \multicolumn{2}{|l|}{2} & 22.2 & & \\
\hline & no & \multicolumn{2}{|l|}{7} & \multicolumn{2}{|l|}{77.8} & \\
\hline
\end{tabular}

Table(4)Sociodemographic distribution of non-user

\begin{tabular}{|c|c|c|c|c|c|}
\hline \multicolumn{2}{|c|}{ Characteristic } & Group & frequency & percent & total \\
\hline \multirow{3}{*}{\multicolumn{2}{|c|}{ Age }} & Up to 40 years & 1 & 1.5 & \multirow{20}{*}{66} \\
\hline & & $41-60$ years & 46 & 69.7 & \\
\hline & & More than 60 years & 19 & 28.8 & \\
\hline \multirow{2}{*}{\multicolumn{2}{|c|}{ Gender }} & male & 51 & 77.3 & \\
\hline & & female & 15 & 22.7 & \\
\hline \multirow{2}{*}{\multicolumn{2}{|c|}{ Residency }} & urban & 56 & 84.8 & \\
\hline & & rural & 10 & 15.2 & \\
\hline \multirow{2}{*}{\multicolumn{2}{|c|}{ Educational level }} & high education & 45 & 68.2 & \\
\hline & & no or primary education & 21 & 31.8 & \\
\hline \multirow{3}{*}{\multicolumn{2}{|c|}{ Occupation }} & employed & 30 & 45.5 & \\
\hline & & worker & 19 & 28.8 & \\
\hline & & No job & 17 & 25.8 & \\
\hline \multirow{2}{*}{\multicolumn{2}{|c|}{$\begin{array}{l}\text { Marital } \\
\text { status }\end{array}$}} & married & 56 & 84.8 & \\
\hline & & single & 10 & 15.2 & \\
\hline \multirow{2}{*}{\multicolumn{2}{|c|}{ Family history of hypertension or other CVD }} & yes & 28 & 42.4 & \\
\hline & & no & 38 & 57.6 & \\
\hline \multirow[t]{2}{*}{ Diabetic } & yes & 32 & \multicolumn{2}{|l|}{48.5} & \\
\hline & no & 34 & \multicolumn{2}{|l|}{51.5} & \\
\hline \multirow{2}{*}{ smoker } & yes & 46 & \multicolumn{2}{|l|}{69.7} & \\
\hline & no & 20 & \multicolumn{2}{|l|}{30.3} & \\
\hline
\end{tabular}




\section{Discussion}

Hypertension causes cardiovascular and renal. Thus decreasing blood pressure reducing morbidity and mortality. The effect of aspirin on control of BP is still a matter of debate ${ }^{(10)}$. Regarding the use of aspirin in healthy or apparently healthy people as primary prevention, there are no direct proofs of its efficacy, but in low-risk populations it may be of no benefit or may be risky ${ }^{(7)}$. In 2016, the (USPSTF) recommendations about aspirin use in primary CVD prevention in has been updated . (11).They adopted a calculator derived (ACC/AHA) for assessment of 10-year risk for first hard atherosclerotic CVD event (non-fatal MI,CHD, death, and fatal or nonfatal stroke) ${ }^{(12)}$.

The study dealt with patients sociodemograhpic characteristics and its association with the habit of aspirin use. The age and the use of aspirin was significantly associated, in which aspirin use was more with increasing age, in those whose ages more than 60 years (120 patients) 97 of them taking aspirin $p$ value 0.003. In a study conducted in Canada that prospectively enrolled 3015 patients without any previous CVS diseases for aspirin use as primary protection, when compared with their counterparts, the study found that , peoples receiving aspirin were older their ages in years was $61.6 \pm 8.4$ vs. $55.5 \pm 8.1$ with $(\mathrm{P}<0.0001)^{(13)}$

A significant association was also found between gender and use of aspirin in which aspirin use was more in females patients with $\mathrm{p}$ value is 0.04 . A similar results was found by a study of mendy et al in USA on the aspirin use in primary protection in patients without prior CVS diseases, they found that aspirin use was more in female $(45.9 \%)$ than in male $(39.1 \%)^{(14)}$.This may because of female in our communities look for and afraid more about their health status. The study found that there was no significant differences between educational level, economic status, residency and occupation with the use of aspirin. $\mathrm{P}$ value $>0.05$.

The study evaluate the relationship of the effect of the score value of the patients and the indication of aspirin use according to score. From the total number of the patients taking aspirin , $19.2 \%$ of them regarded as a misuse of aspirin, which include $17.6 \%$ whose score is below 10 and their age is more than 40 years, and $1.6 \%$ include patients with age below 40 years that is age not assessed by the score. $80.8 \%$ of patients taking aspirin according to score. These findings were approximately similar to a study done by Vanwormer and his associates was done to find

the relationship between; specific sociodemographic variables, and aspirin use among a representative sample of Wisconsin adults without CVD, and found that the percentage of regular aspirin intake in those without indication to take it was 18\% (102 patients from the $\mathbf{5 6 3}$ patients) ${ }^{(15)}$.

This study evaluated the distribution of patient who take aspirin prescribed by their doctors and their risk score was less than $10 \%$. Women were more than men and age between $40-60$ years most of them were urban with medium to higher education half, of them had positive family history of cardiovascular diseases. In a study done by Vanwormer and his associates, they concluded that Aspirin intake was less by patients at high CVD risk which may have a cardiac protection effect from regular use of aspirin and excessive use in patients at low CVD risk. (15). These findings may be due to our patients themselves not complying with their following physician instruction or visiting not a specialist medical physicians with limited knowledge about the risk assessment. So to make any decision for aspirin prescription should fellow an individual clinical judgment that balance the benefits of cardio protection and bleeding risk. Patients with a higher CVD risk, have a greater benefit from aspirin. This final judgment to start aspirin therapy continuously should be made by health care personnel's, especially Primary Care Physicians (PCPs) in resource-poor areas, and they must have a knowledge about their role in assessing the benefit and risk of bleeding. ${ }^{(16)}$

The study show the distribution of patient who take aspirin. which included 5 patients their risk score was less than $10 \%$, and 4 patient had not included in the score whose age below 40 years. Male were predominant live in urban area, all of them were highly educated employed, also most of them had history their family of CVD, this is may be due to educated people be more aware about cardiovascular diseases. In the study of roth et al, performed in an urban county African -Americans and Hispanic adults where the quality of health care delivery should be high, they found that aspirin intake 
was insufficient with high risk patients for CVD and routinely used by many patients at low CVD risk ${ }^{(17)}$.

This study shows the distribution of patient who need aspirin but they didn't use aspirin, they were 66 patients mostly male live in urban area.

higher education, smoker, most of them had no family history of cardiovascular diseases. In a study done by Im et al which was conducted for assessment of the 10-year (CVD) risk and to set for the application of the current requirement on aspirin use for primary protection in Korean participants, and according to 2016 recommendation of (USPSTF), aspirin in their study was indicated in 266 participants $(23.6 \%)$, but only 44 participants of them (3.9\%) were on regular intake of aspirin . Among these who participates in their study, aspirin was prescribed in proper way in $36 \%$ of the participants, suggesting that only $6 \%$ of the participants were taking aspirin appropriately and $3.3 \%$ of them were taking aspirin inappropriately ${ }^{(18)}$

Ethical Clearance: The Research Ethical Committee at scientific research by ethical approval of both environmental and health and higher education and scientific research ministries in Iraq

Conflict of Interest: The authors declare that they have no conflict of interest.

Funding: Self-funding

\section{References}

1. Newby DE, Grubb NR, Bradbury R. Cardiovascular diseases .In : Walker B R, Colledge E R, Ralston $\mathrm{S} \mathrm{H}$ et al. Davidson's Principles and practice of medicine. 22nd Ed .Churchill Livingstone: Elsevier. 2014 ; 607-613.

2. Stone NJ, Robinson JG, Lichtenstein AH, et al . ACC/AHA guideline on the treatment of blood cholesterol to reduce atherosclerotic cardiovascular risk in adults: a report of the American college of cardiology/American heart association task force on practice guidelines. Circulation. 2013; 129 :S1S45.

3. Millard MA, Hernandez-Vila EA . What Do the Guidelines Really Say About Aspirin?. Tex Heart Inst J. 2018 ; Aug 45(4): 228-230.

4. Baigent C, Blackwell L, Collins R et al . Aspirin in the primary and secondary prevention of vascular disease: collaborative meta-analysis of individual participant data from randomized trials. Lancet. 2009 ;373(9678):1849-60

5. Matthys F, DeBacker T, DeBacker G, et al. Review of guidelines on primary prevention of cardiovascular disease with aspirin: how much evidence is needed to turn a tanker? . Eur J Prev Cardiol. 2014; 21: 35465.

6. Lozano R, Franco M E. Which people should take aspirin for primary prevention of cardiovascular disease?. Ther Clin Risk Manag.2015 ; 11: 1043 1045.

7. Coccheri S. Use and Misuse of Aspirin in Primary Cardiovascular Prevention. Clin Med Insights Cardiol. 2017 ; Apr: 21,(11).

8. Van Venrooij, Stolk RP, Banga JD, et al. Primary and secondary prevention in cardiovascular disease: an old-fashioned concept. J Intern Med. 2002 ; Apr 251(4):301-6

9. Assessment of 2013 AHA/ACC ASCVD risk scores with behavioral characteristics of an urban cohort in India, Preliminary analysis of Noncommunicable disease Initiatives and Research at AMrita (NIRAM) study; Medicine (Baltimore) . 2016; Dec 95(49): e5542.

10. Costa AC, Reina-Couto M, Albino-Teixeira A, et al. Aspirin and blood pressure: Effects when used alone or in combination with antihypertensive drugs. Rev Port Cardiol. 2017 ; Jul - Aug 36(78):551-567.

11. Bibbins-Domingo K. Aspirin Use for the Primary Prevention of Cardiovascular Disease and Colorectal Cancer: U.S. Preventive Services Task Force Recommendation Statement. Ann Intern Med.2016; Jun 21; 164(12):836-45.

12. GoffJr D. C, Lloyd-Jones D.M , Bennett G, et al. 2013 ACC/AHA Guideline on the Assessment of Cardiovascular Risk. Circulation.2014 ;129:S49S73.

13. Gupta M, Szmitko PE, Kajil M, et al. Acetylsalicylic acid use in primary prevention in Canada: Insight from the Primary Care Audit of Global Risk Management (PARADIGM) study. Curr Res Cardiol. 2016 ;3(3):67-70.

14. Mendy VL, Vargas R, Zhang L . Aspirin for Prevention of Cardiovascular Disease. Prev Chronic Dis. 2017 ;14:170-171. 
15. Vanwormer J J, Greenlee R T, McBride P E, et al. Aspirin for primary prevention of CVD: Are the right people using it?. J Fam Pract. 2012; September;61(9):525-533.

16. Hennekens CH, DeMets DL. Prevention: aspirin in primary prevention needs individual judgments. Nat Rev Cardiol. 2014 ;11:438-40.
17. Roth G A, Gillespie C W, Mokdad A A, et al. Aspirin use and knowledge in the community: a population- and health facility based survey for measuring local health system performance. BMC Cardiovasc Disord. 2014 ; 14: 16.

18. Im J H, Han S W, Lee S Y, et al. Adherence to guidelines: primary prevention with aspirin in 1125 medical check-up participants. Vessel Plus. 2018 ;2:5. 